Review Article

\title{
Obesity-Related Asthma: Immune Regulation and Potential Targeted Therapies
}

\author{
Yuze Yuan, ${ }^{1}$ Nan Ran $\mathbb{D}^{1},{ }^{1}$ Lingxin Xiong $\mathbb{D}^{1,2},{ }^{1,2 u o q i a n g}$ Wang, ${ }^{1}$ Xuewa Guan, ${ }^{1}$ Ziyan Wang, \\ Yingqiao Guo, ${ }^{1}$ Zhiqiang Pang, ${ }^{1}$ Keyong Fang, ${ }^{1}$ Junying Lu, ${ }^{1,3}$ Chao Zhang, \\ Ruipeng Zheng, ${ }^{1,4}$ Jingtong Zheng ${ }^{\circledR},{ }^{1}$ Jie Ma ${ }^{\circledR},{ }^{2}$ and Fang Wang $\mathbb{D}^{1}$ \\ ${ }^{1}$ Department of Pathogeny Biology, College of Basic Medical Sciences, Jilin University, Changchun 130021, China \\ ${ }^{2}$ School of Pharmaceutical Sciences, Jilin University, Changchun 130021, China \\ ${ }^{3}$ Department of Intensive Care Unit, First Hospital of Jilin University, Changchun 130021, China \\ ${ }^{4}$ Department of Interventional Therapy, First Hospital of Jilin University, Changchun 130021, China
}

Correspondence should be addressed to Jie Ma; ma_jie@jlu.edu.cn and Fang Wang; wf@jlu.edu.cn

Received 20 October 2017; Revised 24 March 2018; Accepted 6 May 2018; Published 28 June 2018

Academic Editor: Hector Rodriguez Cetina Biefer

Copyright (c) 2018 Yuze Yuan et al. This is an open access article distributed under the Creative Commons Attribution License, which permits unrestricted use, distribution, and reproduction in any medium, provided the original work is properly cited.

\begin{abstract}
Obesity, one of the most severe public health problems of the 21st century, is a common metabolic syndrome due to excess body fat. The incidence and severity of obesity-related asthma have undergone a dramatic increase. Because obesity-related asthma is poorly controlled using conventional therapies, alternative and complementary therapies are urgently needed. Lipid metabolism may be abnormal in obesity-related asthma, and immune modulation therapies need to be investigated. Herein, we describe the immune regulators of lipid metabolism in obesity as well as the interplay of obesity and asthma. These lay the foundations for targeted therapies in terms of direct and indirect immune regulators of lipid metabolism, which ultimately help provide effective control of obesity-related asthma with a feasible treatment strategy.
\end{abstract}

\section{Introduction}

Obesity is defined as an excess of body fat, and it is one of the main public health challenges worldwide. It increases the risk for certain diseases and disorders, including type 2 diabetes, hypertension, chronic kidney disease, cardiovascular diseases, certain types of cancer, and depression [1]. According to a previous report, approximately $13 \%$ of adults worldwide are obese [2]. In 2011-2014, 17\% of people aged 2 to 19 years in the US were obese, and in 2011-2012, 38\% were either overweight or obese; these are substantial increases in the past three decades [3]. Obesity in the US accounts for up to one-third of total mortality. A study from the Global Burden of Disease (GBD) revealed that the global obesity epidemic is worsening and is placing heavy public health and economic burdens in most regions of this planet. Thus, effective treatments for controlling obesity is necessary $[1,4]$.
Asthma is a chronic inflammatory disease characterized by variable symptoms of wheezing, shortness of breath, chest tightness, and/or cough and by variable expiratory airflow limitation. It is triggered by multiple factors such as exercise, allergen or irritant exposure, change in weather, or viral respiratory infections [5]. Previous studies have shown that obesity increases the severity of asthma, which demonstrates the close association between these two conditions $[6,7]$. Global epidemiological studies on asthma and obesity have also shown that obesity-related asthma has reached an alarming level [8]. Asthma in obese patients is poorly controlled using standard asthma medications including oral corticosteroids, which is partial because the underlying metabolic mechanism, immune cells, and proteins involved in related signaling pathways may be unresponsive to corticosteroids [9]. Hence, additional treatments are urgently needed for the treatment of obesity-related asthma. 
We have not yet reached a consensus concerning the accurate and comprehensive pathogenesis of obesity. However, lipid metabolism, which is a major part of energy homeostasis, is undoubtedly involved in the onset and development of obesity [4].

In this review, we summarize the association among lipid metabolism, obesity, and asthma. We also detail the roles of immune responses in lipid metabolism and the pathogenesis of obesity-related asthma. Ultimately, we propose various potential targeted therapies according to distinct cellular types and proteins involved in the regulation of lipid metabolism in obesity-related asthma.

\section{Immune Modulators of Lipid Metabolism in Obesity}

2.1. Sterol Regulatory Element-Binding Proteins and Lipid Metabolism. Sterol regulatory element-binding proteins (SREBPs) are significant transcription factors that regulate lipid biosynthesis [10]. This keeps the balance of cholesterol and fatty acids through the activation of gene-encoding enzymes [11]. Additionally, SREBPs play an important role as an interchange node within global signaling networks in a variety of physiological and pathophysiological processes [12]. SREBPs also improve the gene expression of lowdensity lipoprotein (LDL) receptors (LDLR), which are involved in sterol regulation [13-15]. SREBPs are divided into different isoforms, including SREBP1a, SREBP1c, and SREBP2. The physiological roles of SREBPs vary. SREBP1a is involved in global lipid synthesis and growth, SREBP1c is involved in fatty acid synthesis and energy storage, and SREBP2 is involved in the regulation of cholesterol synthesis [16]. Moreover, SREBPs are involved in a myriad of cellular processes and pathologies such as reactive oxygen species (ROS) production, endoplasmic reticulum stress, apoptosis, and autophagy [17]. Nevertheless, the underlying molecular mechanisms remain unclear and need further studies. SREBP1a might modulate the innate immune responses of macrophages, whereas SREBP2 is associated with cell phagocytosis and autophagy, indicating the significant role of SREBPs in the onset and development of chronic inflammatory diseases such as obesity [18-20]. These findings also suggest that targeting SREBPs may be clinically feasible and promising in the treatment of obesity.

2.2. Dipeptidyl Peptidase-4 and Lipid Metabolism. Dipeptidyl peptidase-4 (DPP-4), also known as T-cell surface marker CD26, is widely expressed in multiple cells, particularly in immune cells [20]. It cleaves various chemokines and peptide hormones involved in the regulation of immune response, and it plays an important role in the pathogenesis of inflammation [21, 22]. Previous studies reported on the function of DPP-4, which serves as a surface protease in T-cell activation [23]. Rufinatscha et al. found that in DPP-4 knockdown cells, the levels of triglyceride and peroxisome proliferatoractivated receptor alpha $(\operatorname{PPAR} \alpha)$ were increased, while SREBP-1c expression was obviously decreased [24]. Similarly, Mulvihill found that inhibition of DPP4 significantly reduced postprandial lipoprotein secretion [25]. For example, one selective DPP-4 inhibitor, vildagliptin, led to a significant reduction in total triglyceride and apolipoprotein B-48 (apoB48) concentrations after a high-fat meal [26]. Recent studies showed that another DPP-4 inhibitor, anagliptin, could significantly decrease the expression level of SREBP2 messenger ribonucleic acid, which significantly decreased the plasma total cholesterol and triglyceride levels in anagliptintreated mice. Both low-density lipoprotein cholesterol and very low-density lipoprotein cholesterol levels were also decreased significantly [27].

2.3. Nuclear Factor- (Erythroid-Derived 2-) Like2 and Lipid Metabolism. In addition to SREBP and DPP-4, nuclear factor(erythroid-derived 2-) like2 (Nrf2), a basic leucine zipper transcription factor, is widely expressed in human and mouse tissues as a defense against exogenous and endogenous stimulation [28]. Obesity is a low-grade inflammatory disorder, and in a previous investigation, Nrf2 was reported to be involved in antiobesity activity. Nrf2 inducers mitigated the weight gain, insulin resistance, oxidative stress, and chronic inflammation induced by a high-fat diet (HFD) in mice [29]. In the same study, the weight-reducing and insulin-sensitizing effects of Nrf 2 inducers were abrogated in Nrf $2^{-1-}$ mice, which indicates the importance of Nrf2 in host antiobesity activity. Consistent with the findings of this study, the Nrf2 inducer glucoraphanin increased energy expenditure, decreased lipid peroxidation, and activated M1-like macrophage accumulation and inflammation signaling in HFD-fed mice. Further experiments uncovered that glucoraphanin mitigates obesity by promoting fat browning, limiting metabolic endotoxemiarelated chronic inflammation, and regulating redox stress, which suggests that Nrf2-targeted therapy may be clinically promising in the treatment of obesity [29].

2.4. Intestinal Microbiota and Lipid Metabolism. Bacterial bile salt hydrolase (BSH) enzymes in the gut play a significant role in the metabolism of bile acids [30]. Joyce et al. found that weight gain of mice with normal microbiota and the level of serum cholesterol and liver triglycerides are reduced by the expression of $\mathrm{BSH}$, which indicated that using $\mathrm{BSH}$ may regulate host lipid metabolism [31]. A study showed that the levels of plasma triglycerides and muscle lipid (triglycerides and phospholipids) were significantly decreased in mice fed a diet with prebiotic compared to those that were fed a control diet. Additionally, they also found that the expression of muscle lipoprotein lipase mRNA increased in mice with prebiotic treatment, which may have resulted in the decrease in levels of plasma and muscle lipid [32]. Many studies have demonstrated that HFD feeding profoundly affects the gut microbial community [33-35]. Marc Schneeberger's research indicated that the number of Akkermansia muciniphila was reduced in mice with HFD treatment, which was significantly and positively correlated with fatty acid oxidation and browning. However, it was negatively associated with lipid synthesis, adiposity, and inflammatory markers [36]. The main route of cholesterol excretion is the conversion of cholesterol into bile acid in the liver, followed by excretion of bile acids through the feces. Bile is also considered to be bacteriostatic and to prevent overgrowth of small intestinal bacteria [37]. 
Control bacterial fermentation of dietary fiber in the colon produced short-chain fatty acids (SCFAs). SCFAs regulated the proliferation and apoptosis of cells, which affected intestinal permeability. Additionally, SCFAs could regulate anti-inflammatory effects on the intestinal epithelium by serving as ligands for a series of G-protein-coupled receptors (GPRs) [38]. Experiments have shown that SCFA levels are higher in feces of obese (ob/ob) mice and obese human subjects, which may be due to the reduction of colonic absorption of SCFA leading to obesity [39].

\section{Obesity and Asthma}

In recent years, the incidences of obesity and asthma have been rising with a parallel relationship. The presence of obesity increases the risk for several diseases including asthma [40-42]. Asthma is more common in obese than in nonobese people [43]. Studies have demonstrated that people with asthma have higher BMIs than those without asthma. [44] Moreover, some obese patients with asthma have significant respiratory symptoms and little eosinophilic airway inflammation. Similarly, concordant and growing evidence also supports the relationship between being overweight (defined by body mass index (BMI)) and having asthma [40-42]. Obesity has emerged as a serious risk factor for bronchial asthma, which indicates that obesity could cause or even worsen asthma. Additionally, asthma is more difficult to control in obese patients [45]. Therefore, this study focused on obesity-related asthma. Potential factors that affect the pathogenesis of obesity-related asthma are also summarized in the review (Figure 1).

3.1. Low-Grade Inflammation and Asthma. Obesity is regarded as an inflammatory disease [46]. Unlike typical inflammation, it is chronic low-grade systemic sterile inflammation that is characterized by only moderate upregulation of circulating proinflammatory factors and the absence of clinical symptoms of inflammation [47]. The inflammatory response may affect pulmonary function and thus worsen the asthma. In particular, macrophages play an important role in the occurrence and development of obesityassociated asthma. The HFD mice in obesity modelling increase the number of macrophages in the lungs and alveoli. Besides total cell count in bronchoalveolar lavage fluid (BALF), neutrophils and a few eosinophils also had increased counts [48]. In addition, the concentration of Th1 cytokines and IFN- $\gamma$ also increased significantly in BALF [49]. In healthy adipose tissue, immune cells normally consist of CD4+ T-cell, regulatory T-cells (Treg), and type 2 macrophages (M2), which can regulate heat production, inflammation, and lipid metabolism. Nevertheless, in obese individuals, adipocyte hypertrophy and cytokine secretion result in a shift from M2 to M1 [50] and from Th2 to three different types: Th1, Th17, and CD8+ CTL. Obesity leads to increasing expression of the following: proinflammatory cells such as macrophages; integrins such as CD11b and CD11c; cytokines including TNF- $\alpha$, IL-6, and nitric oxide synthase 2 (NOS2); and triggers such as Toll-like receptors (TLRs), metabolic endotoxemia, lipid spillover, and adipokines. These result in a shift from anti- inflammatory M2 type to proinflammatory M1 type [51]. The activation of NF- $\kappa$ B pathways caused by these cytokines then ensues, thereby inducing the overexpression of proinflammatory cytokines such as TNF- $\alpha$ and IL-6. The activation of adipose tissue macrophages (ATMs) is an amplification of the inflammatory process [52]. In obese humans, long-term nutritional excess can lead to adipose tissue hypertrophy, adipose tissue vascularization, hypoxia, and adipose tissue necrosis. It can also lead to the infiltration of macrophages into adipose tissue and the surrounding necrotic tissue, which also produces a wide variety of proinflammatory cytokines [53]. In addition, the secretion of inflammatory cytokines and activation of the NF- $\kappa \mathrm{B}$ pathway trigger transcriptional expression of Nod-like receptor family pyrin domain containing 3 (NLRP3) and pro-IL-1 $\beta$ as well as the subsequent activation of NLRP3, causing macrophages to produce IL- $1 \beta$. Overproduced saturated fatty acids in obese individuals can also stimulate NLRP3 inflammasome activation [54]. Inflammatory factors spread from adipose tissue into the blood circulation and then reach the lungs, which trigger airway inflammation and hyperresponsiveness. It has been reported that TNF- $\alpha$ can directly induce airway hyperresponsiveness (AHR) [55]. In addition, obesity can lead to abnormal fatty acid metabolism and increased fatty acid in blood by promoting increased expression of ACC1 and subsequently activation of ROR $\gamma$ t to induce the differentiation of Th17 cells. IL-17 is produced by Th17 cells that bind with its receptors. IL-17 is able to improve the secretion of inflammatory cytokines such as IL- 6 , TNF- $\alpha$, IL-8, CAM-1, and CM-CSF through the MAPK or NF- $\kappa$ B pathway [56]. The association between obesity and neutrophil count in sputum is significant; in addition, a recent cluster analysis has shown that the presence of obesity-related asthma is characterized by increased airway neutrophils [57-59]. In addition to this, IL-17, IL-6, and TNF- $\alpha$ secreted by Th17 cells recruit and activate neutrophils in the lungs [60]. Neutrophils play an important role in ATM recruitment and inflammation by degradation of insulin receptor substrate 1 or activation of the TLR-4 pathway [61]. In addition to Th17 cells, Th1 cells in abnormal adipose tissue of obese individuals can secrete cytokines including IL- 2 , IL-3, IFN- $\gamma$, and TNF- $\alpha$, which may activate $\mathrm{M} 1$ and thereby activate neutrophils through the chemokines (C-X-C motif) ligand 8 (CXCL8) pathway. Obesity-associated AHR was independent of adaptive immunity. A few eosinophils but many neutrophils were found in sputum, which suggests that obesity-associated asthma may be allergen independent [9]. Apart from the immune responses mentioned above, innate lymphoid cells also participate in the pathogenesis of obesity-related asthma. Although ILC2s have been reported to promote AHR and airway inflammation in a previous study [62], other studies showed that ILC2s played a crucial role in the repair of the airway epithelium, metabolic regulation, and translation from white adipose tissue into beige adipose tissue in the lungs [63]. However, a lack of ILC2 protection in obesity-related asthma may be partially due to the replacement of the ILC2 response with ILC3 response. In a study involving diet-induced obese mice, ILC3s and Th17 as well as the corresponding release of IL-17 were observed in the BALF, which may promote the development of AHR [64]. Bronchial epithelial cells stimulated by 


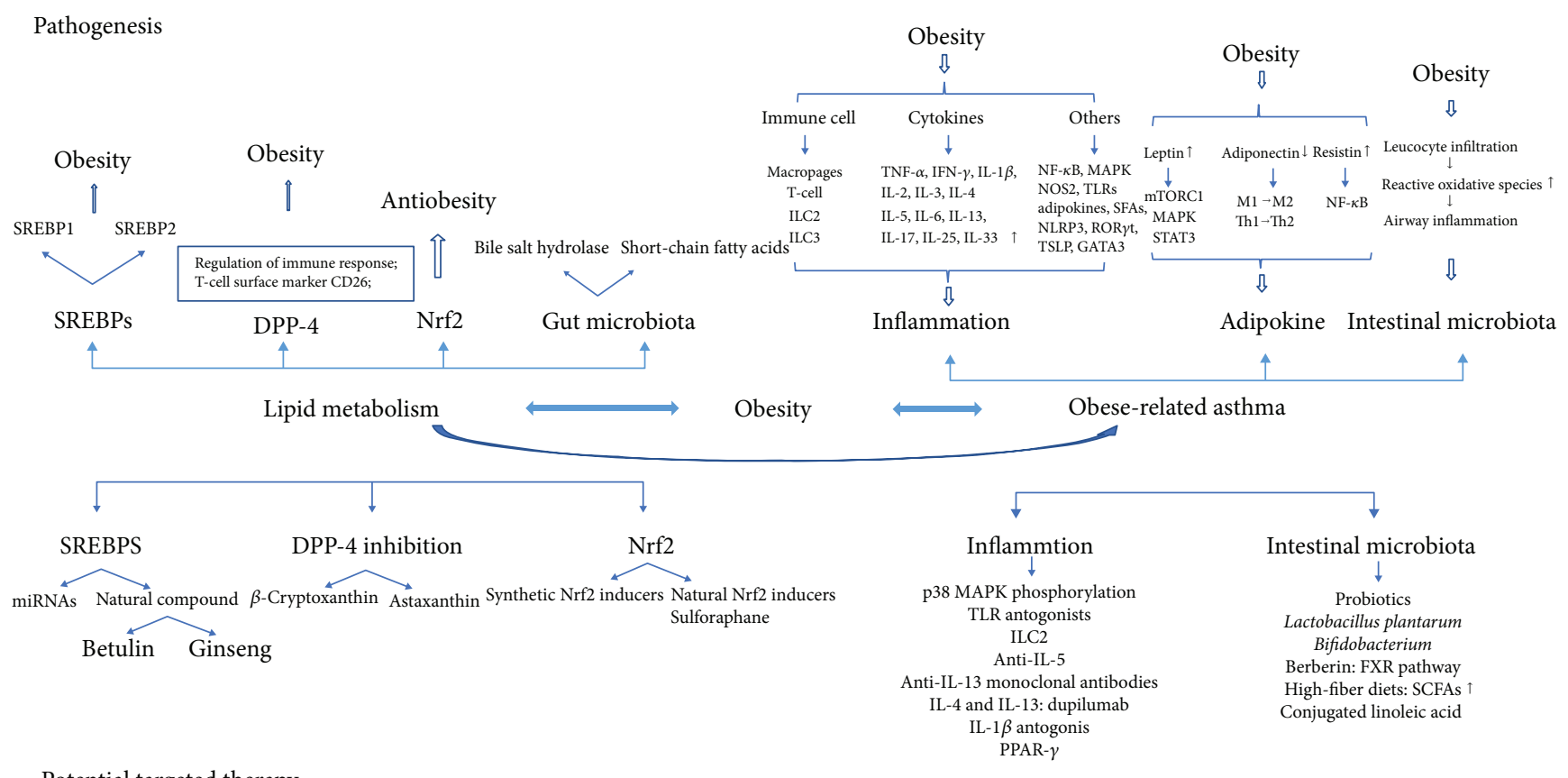

Potential targeted therapy

Figure 1: Possible connections among lipid metabolism, obesity, and obesity-related asthma. This figure is divided into two parts: the upper part about pathogenesis and the lower part about potential targeted therapy. The part on pathogenesis demonstrates a few major effects of immune regulators. The other introduces the corresponding possible targeted therapy including SREBPs, miRNAs, and the DPP-4 inhibitor.

exogenous substances such as ozone [65] or cytokines can secrete IL-25, IL-33, and thymic stromal lymphopoietin (TSLP), thereby activating ILC2 to produce type 2 cytokines such as IL-4, IL-5, and IL-13. The pathway depends on the activation of the transcription factor GATA3 [66]. Macrophages, neutrophils, ILC2, Th1, and Th17 secrete a wide variety of cytokines and chemokines, which act on bronchial smooth muscle and may subsequently cause a series of asthmatic symptoms including airway narrowing, airway remodeling, mucus hypersecretion, AHR, airway smooth muscle constriction and hypertrophy, and a rapid decline in lung function.

3.2. Adipokine Secretion and Asthma. Obesity-related lowgrade inflammation originates from the adipose tissue, which enables the secretion of a variety of interleukins and adipokines such as leptin, adiponectin, and resistin. In turn, these factors can affect obesity-related inflammation and airway inflammation [67]. Therefore, adipose tissue can be considered as a typical endocrine organ [68]. The amount of leptin produced by adipose tissue is higher in obese subjects compared to their nonobese counterparts [69]. However, a previous study found that leptin function in obese patients did not change. Leptin tolerance may be a causative factor in obesityrelated asthma [70]. Moreover, overproduced leptin can stimulate the production of proinflammatory mediators, such as TNF- $\alpha$, IL- 6 from the adipose tissue, and IFN- $\gamma$ from Th1 immune responsive CD4+ T-cells. TNF- $\alpha$ and IFN- $\gamma$ are the mediators associated with AHR in asthma. On the other hand, leptin may inhibit the function and proliferation of regulatory $\mathrm{T}$-cells, which may impair the balance of Th1 and Th2 and promote the polarization of Th1-mediated autoimmune diseases and Th2-mediated immune diseases such as asthma [71, 72]. Leptin not only affects the innate immunity but also has a significant impact on the allergic inflammatory response in obese individuals. Leptin promotes the proliferation and survival of proallergic Th2 cells and ILC2 by activating mTORC1, MAPK, and STAT3 pathways, which leads to the production of type 2 cytokines such as IL4, IL-5, and IL-13, which together contribute to allergic responses [73]. In short, IL-4 promotes the production of IgE and subsequently activates mast cells; IL-5 activates and recruits eosinophils. IL-13 can directly act on goblet cells, which results in the secretion of airway mucus, airway remolding, and AHR [74]. A study found that the release of airway mucus induced by IL-13 can be regulated through the JAK2-STAT3-MUNC18b pathway [75]. There is no evidence that leptin is a direct cause of asthma. However, several studies have demonstrated that leptin is correlated with obesity and asthma in both adults and children $[69,76,77]$. Adiponectin is an anti-inflammatory adipokine produced by adipose tissue [78]. Adiponectin participates in inflammation by downregulating proinflammatory cytokines including TNF$\alpha$, IL-6, and NF- $\kappa \mathrm{B}$ and upregulating anti-inflammatory cytokines such as IL-1 receptor antagonists and IL-10. In addition, the decreased level of adiponectin reduces the inhibitory effect of bacterial lipopolysaccharide (LPS), thereby inducing macrophages to produce IFN- $\alpha$, which induces the body's lowgrade inflammatory reaction [79]. Adiponectin also induces a shift from M1 to M2 and from Th1 to Th2 and enhances the immunity and anti-inflammatory ability of body [80]. However, despite the enhanced inflammation in obese 
individuals, their levels of adiponectin are low [46]. This may result in the decreased anti-inflammatory activity of adiponectin. In addition, the overexpression of proinflammatory cytokines may inhibit the secretion of adiponectin, which results in further reduction in the anti-inflammatory effect of adiponectin, thereby providing the appropriate immunological environment for the onset and development of asthma. Furthermore, the effect of distinct isoforms of adiponectin varies in obesity-related asthma cases. A recent study found that the higher concentration of low-molecular-weight (LMW) adiponectin and the lower ratio of middle molecular weight (MMW) adiponectin/total adiponectin were evidently associated with asthma [81]. However, the level of high-molecularweight (HMW) adiponectin in serum is not related to the onset and development of obesity-associated asthma [67]. Resistin, like leptin, is another proinflammatory adipokine produced by adipose tissue. Obesity is a chronic low-grade inflammatory condition accompanied with the increased production of various inflammatory factors including resistin. Moreover, inflammatory factors such as IL-1, IL6 , TNF- $\alpha$, and LPS can promote resistin expression via NF- $\kappa$ B-induced pathways. In turn, the proinflammatory cytokines including IL- 6 and TNF- $\alpha$ can be promoted by resistin, thus decreasing obesity-associated inflammation [82]. These proinflammatory factors act on the lungs, which may lead to increased airway inflammation and asthma [83]. Through the comparison of serum leptin levels, Hassan et al. found that obese subjects with asthma showed higher resistin levels. With the increase in resistin levels, asthma severity is also accordingly increased [83]. Similarly, other studies have also demonstrated that the level of resistin and resistin/adiponectin ratio are proportionally increased in asthma and are even higher in obese subjects with asthma. In addition, the level of resistin and resistin/adiponectin ratio can also negatively predict lung function [67]. The above studies suggest that resistin can aggravate inflammation and promote the onset and deterioration of asthma. Thus, obesity may promote the occurrence of asthma and further aggravate asthma by promoting the increase of resistin. These studies probably uncovered a novel therapeutic target for obesity-related asthma.

3.3. Intestinal Microbiota Dysbiosis and Asthma. Intestinal microbiota mainly act as a biological barrier and are involved in immune regulation. Bacterial diversity increases mucosal immune defense [84]. However, obesity may result in intestinal microbiota dysbiosis and the reduction of bacterial diversity with an increase in Firmicutes and a reduction in Bacteroidetes [85], which are responsible for intestinal barrier and immune function damage. Thus, this results in weight gain, systemic inflammation, insulin resistance, and asthma [86]. Studies have reported that the concentration of LPS in plasma increased significantly in obese individuals, which may be due to the increase in intestinal permeability and excessive HFD [87]. Obesity-related low-grade inflammation and intestinal microbiota disorders can cause increased intestinal mucosal permeability [88]. LPS moves from the intestinal mucosa into the blood circulation, leading to endotoxemia. The related underlying mechanism is as follows: binding of LPS with TLR4 may activate the NF- $\kappa$ B pathway, which produces a wide variety of cytokines, including TNF- $\alpha$ and IL-6 [89]. These cytokines act on the lungs, which may result in AHR and asthma exacerbation. Diet also affects the intestinal microbiota, thereby influencing asthma. A high-fiber/low-fat diet can increase the circulating levels of SCFAs [87] that play a critical role in inhibiting inflammation by regulating the differentiation and activation of Treg [90, 91], inhibiting LPS-induced NF- $\kappa$ B activation, increasing TNF- $\alpha$ levels in neutrophils and macrophages, and inhibiting neutrophil production of proinflammatory reactive oxygen species (ROS) and TNF- $\alpha$ [92]. SCFAs stimulate intestinal epithelial proliferation and differentiation and could also contribute to the repair of the epithelial cell damage that is typical in asthma [92]. Moreover, SCFAs produced by the metabolism of dietary fiber from gut bacteria also promote the secretion of leptin to inhibit weight gain. However, a high-fat/low-fiber diet results in decreased levels of SCFAs and the imbalance of gut bacteria [93]. A study on an animal model reported that a low-fat/high-fiber diet promoted the production of beneficial bacteria such as Lactobacilli, Bifidobacteria, and Faecalibacterium and the production of SCFAs, especially butyrate. In contrast, the high-fat/low-fiber diet increased the Enterobacteriaceae, which are harmful to human health conditions [94]. High-fat/low-fiber dietinduced dysbacteriosis inhibits the function of regulatory T-cells through epigenetic modifications of the Forkhead box P3 (FoxP3) promoter and increases Th2-induced allergic airway inflammation. HFD-induced obesity may increase the risk for asthma via the changing gut bacteria. In addition, the obesity-induced dysbiosis of gut bacteria causes cholesterol metabolic disorders and the levels of bile acids are reduced in the gut, which impair the inhibition of NLRP3. NLRP3 activation induces IL- $1 \beta$ secretion primarily by M1 macrophage, which induces AHR that is considered as a major feature of asthma [95]. Many epidemiological studies have shown that with the increased number of caesarean section deliveries, there has also been an increase in obesity, type 1 diabetes, allergies, celiac disease, and some neurological disorders [96]. Caesarean-born children did not pass through the mother's birth canal, which causes intestinal microbial abnormalities, thus resulting in offspring obesity [97]. Studies found that compared with natural-born mice, caesareanborn mice gained more weight [98]. In addition, women who undergo caesarean section delivery usually use antibiotics preventively, which may be harmful to the baby's intestinal microbiota and may exacerbate obesity and other diseases. Another study found that ovalbumin-induced asthmatic mice had increased diversity of intestinal microbiota when exposed to microbes in early life. Moreover, IFN- $\gamma$ levels and the ratio of IFN- $\gamma$ and IL- 4 were also increased significantly, which suggests that increased diversity of intestinal microbiota may induce Th1 response and inhibit airway inflammation in allergic asthma [99]. The results of these studies, taken together, indicate that a lack of microbial exposure in early life may cause obesity and asthma. Hence, we think that obesity caused by the lack of microbial exposure in early life may result in the risk and deterioration of asthma. 


\section{Potential Targeted Therapy of Obesity- Associated Asthma}

There is a close association between obesity-associated asthma and several well-established risk factors for morbidity; thus, reversing the obesity-associated asthma is an urgent priority. We summarize herein potential targeted therapies for immune regulation, intestinal microbiota, and inflammation to control obesity-associated asthma (Figure 1). A better understanding of these different therapies will lead to future advances in the clinic.

4.1. Immune Regulator-Targeted Therapies. MicroRNAs (miRNAs), a class of noncoding RNAs, emerge as a novel treatment strategy for dyslipidemia and obesity via the modulation of sterols by SREBPs [100]. Among them, the most popular miRNA in the modulation of lipid metabolism is miR-33a, which is located in the intron of SREBF2. miR-33 regulates high-density lipoprotein (HDL) biogenesis and cholesterol efflux by downregulating the expression of ATP-binding cassette transporters [12]. miR-33 also targets SREBP1c, thereby affecting obesity [101]. A study using humanized knock-in mice observed the contribution of miR-33b to a relatively low HDL cholesterol level in human beings [101]. After induction by host SREBF genes, the miR-33 system facilitates lipid homeostasis by modulating the opposing regulator systems (plasma LDL versus HDL cholesterol and cholesterol versus fatty acid metabolism), indicating the therapeutic intervention of miR-33 in dyslipidemia [102]. Another example that has found clinical success in the regulation of lipid metabolism via SREBP is PCSK9 inhibitors, which indicate SREBP2 target PCSK9 in plasma and contribute to LDLR degradation [103, 104].

Intriguingly, extracts and natural compounds derived from some plants are also reported to possess antiobesity activity by targeting SREBP genes and other adipogenesisrelated genes (such as PPAR $\gamma$ and ACC1). Betulin, a natural triterpene isolated from the bark of birch trees, was reported to inhibit the maturation of SREBP by inducing the interaction of SREBP cleavage-activating protein (SCAP) and insulin-induced gene protein (INSIG), which lowered the biosynthesis of cholesterol and fatty acid. In vivo, betulin ameliorated diet-induced obesity by decreasing the lipid contents in serum and tissues and concomitantly enhancing insulin sensitivity. Thus, this is a potential compound for the treatment of hyperlipidemia and obesity [105]. Ginseng, which has been used in traditional Chinese medicine for centuries, is a novel depressor against SREBP and other transcriptional factors such as PPAR $\gamma$ and ACC1. A South Korean research group found that compared with HFD mice, mice receiving HFD with Korean red ginseng extract (GE) $(10 \mu \mathrm{g} / \mathrm{ml})$ for eight weeks had decreased body weight, adipose tissue mass, and adipocyte size, which indicated significant antiobesity effect [106]. Additionally, data in vitro confirmed that GE and two major ginsenosides, Rb1 and Rg1, inhibited adipogenesis by lowering $\operatorname{PPAR} \gamma, \mathrm{C} / \mathrm{EBP} \alpha$, and SCD1 expression at the gene level. Other studies have also reported significant antiobesity activity of Korean red ginseng by modulating transcription factors such as PPAR $\gamma$ and aP2, which decrease the amount of lipid accumulation and inhibit adipogenesis [107-109]. These observations suggested that natural herbs are abundant medical sources for targeting SREBPs and other transcriptional factors in the treatment of obesity-related asthma.

In addition to inhibitors targeting the transcriptional factor SREBP, two independent research groups highlighted the potential clinical application of DPP-4 inhibitors in the attenuation of macrophage cell-mediated or immune cell-mediated inflammation $[110,111]$. DDP-4 inhibitor or carotenoids such as $\beta$-cryptoxanthin and astaxanthin facilitate the immune regulation of lipid homeostasis in vitro at least partly by the decline in M1 macrophage numbers and the increase in M2 macrophage numbers, which indicate potential application in the treatment of obesity-related asthma in clinical and scientific studies $[112,113]$. There was evidence demonstrating the role of Nrf2 in the treatment of obesity. A wide variety of synthetic Nrf2 inducers including triterpnoid 2-cyano-3, 12dioxoolean-1, 9-dien-28-oic acid- (CDDO-) imidazolide, dithiolethione analog, and oltipraz demonstrated a significant ameliorative effect on HFD-induced obesity [114, 115]. However, it seemed that synthetic Nrf2 inducers were not clinically available due to the significantly increased risks of heart failure and the composite cardiovascular outcomes (nonfatal myocardial infarction, nonfatal stroke, hospitalization for heart failure, or death from cardiovascular causes) [116]. Sulforaphane, an isothiocyanate derived from cruciferous vegetables, is one of the most potent naturally occurring Nrf2 inducer. The compound was reported to ameliorate obesity by the enhancement of energy expenditure and the reduction of metabolic endotoxemia, which were caused by the decline in inflammation and insulin resistance. Sulforaphane may be a promising treatment for obesity-related asthma [29].

4.2. Intestinal Microbiota-Targeted Therapies. Gut microbiota have been associated with obesity-activating innate immunity through the LPS Toll-like receptor 4 axis [117]. Gut microbiota dysbiosis may induce obesity-related asthma [118]. We hypothesized that it may be beneficial to improve or treat obesity-related asthma via the modulation of gut microbiota. Moreover, the metabolic products of intestinal microbiota have a significant effect on body metabolism and immune defense mechanisms. Probiotics have a significant effect on improving intestinal microbiota modulation and systemic immunity. Previous studies indicated the weight of mice fed with HFD was the highest in three groups of mice with HFD, mice with HFD plus probiotics, and mice with HFD plus Lactobacillus plantarum (LP). The mice in high-fat diet plus LP exhibited significantly lower IL-6 and endotoxin (ET) content. Moreover, it will preferably regulate intestinal microbiota and systemic immune function to feed the combination of Lactobacillus plantarum (LP) and Lactobacillus fermentum (LF) [119]. In view of systemic obesityrelated inflammation, the use of probiotics in the treatment of obesity probably has a potential effect on improving asthma. Clinical evidence demonstrated that the microbiome of obese people lacked a cluster of abundant Bacteroides species. Animal experiments showed that gavage with $B$. thetaiotaomicron can reduce serum glutamate concentration, which 
increased lipolysis and fatty acid oxidation process, thereby reducing fat accumulation and eventually achieving weight loss. Thus, it may be feasible to prevent obesity by regulating gut microbiota [120].

Obesity-related microbiota dysbiosis influences the production of bile acid, which is associated with lipid digestion and absorption [121]. Intestinal bile acids can be absorbed by bile acid binding resin (BAR) and likely improve obesity and metabolic disorders [122]. The binding of Berberine (BBR), extracted from the roots of Rhizoma coptidis, with gut and intestinal Farnesoid X receptor (FXR) lead to decreasing serum lipids in humans, hamsters, mice, and rats [123]. BBR inhibited bile salt hydrolase (BSH) activity in gut microbiota and activated FXR signaling pathway to alter bile acid metabolism, which may regulate lipid metabolism and then achieve weight loss. Therefore, BBR administration may be an effective strategy to improve obesity-related asthma [124].

Diet can also affect the intestinal microbiota balance and improve obesity and asthma. High-fiber diets can increase intestinal and circulating SCFA concentrations to suppress allergic inflammation and weight gain, which relies on the metabolism of intestinal microbiota [125]. Trompette et al. reported that high-fiber diet and SCFAs can shape immunological environment in the lungs by regulating the differentiation and activation of Treg. They can also affect the severity of allergic inflammation by inhibiting neutrophil production of proinflammatory reactive oxygen species (ROS) and TNF$\alpha$ [90]. Thus, a diet with abundant dietary fermentable fiber may be beneficial for patients with obesity-related asthma. In addition, we summarized that increased activation of microbes producing SCFAs or the direct application of microbes producing SCFAs via fiber metabolism may also improve the symptoms of obesity-related asthma. Moreover, a prospective cohort study indicated that yogurt significantly prohibited weight gain, in particular among participants with higher fruit consumption, which may be due to a mechanism mediated by probiotics such as Lactobacillus and Bifidobacterium [126]. Additionally, gastrointestinal bacteria could metabolize foods such as beef, yogurt, and vegetable oils to produce conjugated linoleic acid (CLA) such as trans-10 and cis-12 CLA, which significantly prevented weight gain via mechanisms that increase adipocyte turnover and lead to the appearance of metabolically active beige adipocytes. Free linoleic acid and $\alpha$-linolenic acid are converted to different CLA by Bifidobacterium, Bifidobacterium pseudolongum strain, and Bifidobacterium breve strains [127]. Therefore, CLA and CLA-associated compounds are a novel strategy to control weight that may be beneficial to improve obesityrelated asthma.

4.3. Inflammation-Targeted Therapies. Obesity-related asthma is a chronic inflammatory disease accompanied with the disorder of proinflammatory and anti-inflammatory molecules driven by cytokines. Therefore, the regulation of cytokine secretion may be a new strategy to inhibit inflammation-induced obese-related asthma. MKP-1, a MAPK deactivator, can produce $\mathrm{p} 38$ MAPK phosphorylation in an irreversible manner, thereby inhibiting the occurrence of inflammation. Prabhala found that binding of dexamethasone or compounds interrupting the proteasome with MKP-1 can induce p38 MAPK phosphorylation and inhibit inflammatory cytokines in airway smooth muscle cells [128]. The interaction of p38 MAPK and MKP-1 may be a novel therapeutic target for obesity-associated asthma. Obesity-associated inflammation is mostly related to TLR4NF- $\kappa B$ pathway [129]. TLR4 knockout or antagonists may be beneficial for obesity-related asthma. TLR4 knockout relieves HFD-induced phosphorylation of IKK $\beta$, JNK, mTOR, and proinflammatory signaling molecules, which can alleviate obesity-associated inflammation. It might implicate the possible therapeutic potential of TLR4 in the management of asthma in HFD-induced obesity [130]. Furthermore, TLR antagonists have been used to treat metabolic diseases due to the beneficial effects of immune suppression in modern medicinal studies and applications. However, HFD-induced low-grade chronic inflammation may be an evolutional protective mechanism against pathogens. TLR antagonists or TLR knockout may inhibit the activity of host TLR, which probably increases the vulnerability to infection [131].

Chronic low-grade inflammation of the body induced by obesity also followed ILC2 activation. Cytokines derived from lung epithelial cells, such as IL-25, IL-33, and TSLP, can activate ILC2 to cause pulmonary injury. The activated ILC2s subsequently produce type 2 cytokines (IL-4, IL-5, and IL-13) and induce severe inflammation in the lungs [73]. Thus, inhibition of IL-25, IL-33, and TSLP possibly relieves type 2 inflammation. In a previous publication, a combination of an anti-TSLP antibody, AMG 157, and TSLP effectively obstructed the interplay between TSLP and its receptor, which may inhibit ILC2 activation [132]. Many clinical trials have revealed that antibodies against IL-5 or IL-5 receptor, IL-13, and IL- $4 \mathrm{R} \alpha$ modestly reduced asthma exacerbations and improved lung function [133]. Antiinterleukin-5 (anti-IL-5) is a neutralizing antibody targeting IL-5, which is essential for eosinophil maturation and survival. Two anti-IL-5 drugs have been approved by the Food and Drug Administration (FDA) in the US: mepolizumab [134], by subcutaneous injection monthly, and reslizumab [135], by intravenous infusion monthly. Ramirez-Carrozzi et al. concluded that commissural inhibition of IL-13 and IL-33 pathway or IL-5 and IL-13 pathway was extremely effective to reduce type 2 inflammation in patients with severe asthma [136]. Of note, the blockade of interleukin13 using the two anti-interleukin-13 monoclonal antibodies, lebrikizumab and tralokinumab, potentially improved airway inflammation and smooth-muscle reactivity, which may reduce FENO but increase circulating eosinophil counts. The growth in peripheral blood eosinophil counts have been reported previously to reflect blocking of IL-13 activity [137]. In 2015 , in a phase II clinical study of using lebrikizumab to patients with moderate-to-severe uncontrolled asthma despite ICS therapy and an additional controller, subcutaneous administration of lebrikizumab taken every four weeks reduced asthma exacerbation rate by $60 \%$ compared with placebo in periostin-high patients and by $5 \%$ in periostinlow patients. In this study, despite improving lung function, 
lebrikizumab treatments have not yet led to clinically meaningful placebo-corrected improvements in asthma symptoms or quality of life, potentially due to the limited power of the studies for these safety endpoints [138]. In 2017, the researchers had phase III clinical studies to provide further evidence of the safety and efficacy of lebrikizumab. Lebrikizumab, targeting IL-13 alone with biologics, has not shown a consistent reduction in asthma exacerbation. However, they confirmed that dupilumab, a medication simultaneously targeting at both IL-4 and IL-13 via blocking IL-4 receptor, has yielded more consistent results in reducing asthma exacerbations and improving lung function, especially in patients with increased blood eosinophils. Therefore, biologics targeting IL-4/IL-13 may be useful in patients with proof of T2-high asthma based on the presence of type 2 inflammation regardless of their baseline blood eosinophil levels [139]. Besides, late phase clinical trials of drugs targeting the IL-4/IL-13 pathways show promising results to achieve FDA-approved therapies. Doherty has said that TSLP can induce partial corticosteroid resistance, but under this condition, corticosteroid can still inhibit IL-33 to activate ILC2 [140]. Therefore, it is possible that corticosteroid can improve modestly obesity-related asthma. In addition, the function of ILC2 can be inhibited by Treg cells by the secreting cytokines of IL-10 and TGF- $\beta$ in adipose tissue or via the direct contact of ILC2 with Treg, which can improve type 2 inflammation and deterioration of lung function. Therefore, taking effective measures to increase the number of Treg cells in asthmatic patients may reduce the inflammatory response induced by type 2 inflammatory cells [141]. In addition to ILC2, a team conducting experiments in mouse models of HFD concluded that NLRP3, IL-1 $\beta$, and ILC3 cells facilitated obesity-related asthma by mediating inflammation [142]. In an experiment, after a short treatment of the IL- $1 \beta$ antagonist, anakinra, the symptom of AHR of obese mice induced by high-fat diets was improved [143]. Another team conducted a phase I clinical study, the result of which was that anakinra effectively reduced airway neutrophilic inflammation and caused no serious adverse events in a model of inhaled endotoxin LPS challenge. Thus, anakinra can be regarded as a potential therapeutic candidate for treatment of asthma with neutrophil advantage [144]. To date, it has not been investigated whether this type of treatment could be clinically applied in humans. In addition to inhibiting inflammatory cytokines, overall anti-inflammatory therapy may also be effective in obesity-related asthma. Similarly, obesity-related asthma frequently accompanies insulin resistance due to the lack of adiponectin. Calixto et al. conducted a study using obese mouse model fed with a HFD and found that metformin, a first-line treatment for diabetes, attenuated the exacerbation of the allergic eosinophilic inflammation [145]. In a retrospective cohort study, metformin users had a lower risk for asthmarelated hospitalization and asthma exacerbation [146]. Hence, healthcare providers should consider metformin as a potential medication for patients with concurrent asthma and diabetes. It is well known that macrophages play an important role in the development and deterioration of obesity-related asthma and that peroxisome proliferatoractivated receptors (PPARs) are expressed in monocytes/ macrophages and adipose tissue. The activation of PPARs inhibits the shift from M2 to M1 and from Th2 to Th1 and also inhibits the secretion of proinflammatory cytokines such as IL-1 $\beta$, IL-6, IL-10, IL-12, and TNF- $\alpha$ [147]. A study conducted by Yoon et al. demonstrated that the activation of PPAR- $\gamma$ induced by apoptotic cell instillation over the course of bleomycin-induced lung injury can reverse the enhanced efferocytosis, the decreased expression of proinflammatory cytokines, and neutrophil recruitment, which likely inhibits inflammatory responses. Moreover, PPAR- $\gamma$ activation may cause specific death of macrophages [148]. Therefore, PPAR- $\gamma$ activation with PPAR- $\gamma$ agonist or other PPAR- $\gamma$ stimulating compounds may reduce obesity-associated inflammation, thereby improving the severity of obesityrelated asthma. To date, PPAR- $\gamma$ agonists have not been studied clinically. Moreover, the regulation of adipokine level in obese individuals can also improve obesity-related asthma. Adiponectin secreted by adipose tissue is an antiinflammatory adipokine and can promote the utilization of intracellular fatty acid and triglyceride-content reduction, whereas the level is decreased in obese individuals [149]. It has been demonstrated that adiponectin resistance observed in obese patients is due to the increased level of adiponectin in serum and the defective expression of adiponectin receptors in the lungs [150]. Despite some studies having shown no effect of recombinant adiponectin in animals, recombinant adiponectin may be a challenging therapeutic strategy for obesity-related asthma in the future.

\section{Conclusion}

As obesity becomes more prevalent worldwide, obesityrelated asthma is frequently observed in the whole population. In this review, we described the disturbed lipid metabolism and immune modulators of lipid metabolism in obesity such as SREBPs, DPP-4, and Nrf2. In addition, we also discussed several immune factors potentially contributing to the pathogenesis of obesity-related asthma including intestinal microbiota, immune regulator, and inflammation. According to these possible immune causes in the onset and development of obesity-related asthma, we summarized several promising targeted therapies in the treatment of obesity-related asthma, such as miRNAs and TLR antagonists, which may provide effective medical intervention strategy in controlling obesityrelated asthma.

\section{Conflicts of Interest}

There is no conflict of interests regarding the publication of this review.

\section{Acknowledgments}

This work was supported by the Fundamental Research Funds for the General Universities (Grant no. 451170306058), Graduate Innovation Fund of Jilin University (Grant no. 2017139), Key Laboratory of Health and Family Planning Commission of Jilin Province of China (Grant no. 3D5172303426), and Provincial School Co-construction Industrialization 
Demonstration Project of Jilin Province (Grant no. SXGJSF2017-1-1(01)). The authors thank Ying Liu for revising the manuscript.

\section{References}

[1] E. W. Gregg and J. E. Shaw, "Global health effects of overweight and obesity," New England Journal of Medicine, vol. 377, no. 1, pp. 80-81, 2017.

[2] B. Gross, M. Pawlak, P. Lefebvre, and B. Staels, "PPARs in obesity-induced T2DM, dyslipidaemia and NAFLD," Nature Reviews Endocrinology, vol. 13, no. 1, pp. 36-49, 2017.

[3] E. A. O'Connor, C. V. Evans, B. U. Burda, E. S. Walsh, M. Eder, and P. Lozano, "Screening for obesity and intervention for weight management in children and adolescents: evidence report and systematic review for the US preventive services task force," Journal of the American Medical Association, vol. 317, no. 23, pp. 2427-2444, 2017.

[4] M. W. Schwartz, R. J. Seeley, L. M. Zeltser et al., "Obesity pathogenesis: an endocrine society scientific statement," Endocrine Reviews, vol. 38, no. 4, pp. 267-296, 2017.

[5] Global Initiative for Asthma, "Global strategy for asthma management and prevention," 2018, http://www.ginathma.org.

[6] P. D. Freitas, P. G. Ferreira, A. G. Silva et al., "The role of exercise in a weight-loss program on clinical control in obese adults with asthma. A randomized controlled trial," American Journal of Respiratory and Critical Care Medicine, vol. 195, no. 1, pp. 32-42, 2017.

[7] H. A. Scott, L. G. Wood, and P. G. Gibson, "Role of obesity in asthma: mechanisms and management strategies," Current Allergy and Asthma Reports, vol. 17, no. 8, p. 53, 2017.

[8] L. G. Wood, "Asthma in the obese: a big and growing problem," American Journal of Respiratory and Critical Care Medicine, vol. 195, no. 1, pp. 4-5, 2017.

[9] D. T. Umetsu, "Mechanisms by which obesity impacts upon asthma," Thorax, vol. 72, no. 2, pp. 174-177, 2017.

[10] Y.-C. Kuan, T. Hashidume, T. Shibata et al., "Heat shock protein 90 modulates lipid homeostasis by regulating the stability and function of sterol regulatory element-binding protein (SREBP) and SREBP cleavage-activating protein," Journal of Biological Chemistry, vol. 292, no. 7, pp. 30163028, 2017.

[11] M. S. Brown and J. L. Goldstein, "A proteolytic pathway that controls the cholesterol content of membranes, cells, and blood," Proceedings of the National Academy of Sciences, vol. 96, no. 20, pp. 11041-11048, 1999.

[12] H. Shimano and R. Sato, "SREBP-regulated lipid metabolism: convergent physiology-divergent pathophysiology," Nature Reviews Endocrinology, vol. 13, no. 12, pp. 710-730, 2017.

[13] S. Rong, V. A. Cortés, S. Rashid et al., "Expression of SREBP1c requires SREBP-2-mediated generation of a sterol ligand for LXR in livers of mice," eLife, vol. 6, 2017.

[14] M. S. Brown and J. L. Goldstein, "The SREBP pathway: regulation of cholesterol metabolism by proteolysis of a membrane-bound transcription factor," Cell, vol. 89 , no. 3 , pp. 331-340, 1997.

[15] L. A. J. O'Neill, R. J. Kishton, and J. Rathmell, “A guide to immunometabolism for immunologists," Nature Reviews Immunology, vol. 16, no. 9, pp. 553-565, 2016.
[16] S. Li, Y.-T. Oh, P. Yue, F. R. Khuri, and S.-Y. Sun, "Inhibition of mTOR complex 2 induces GSK3/FBXW7-dependent degradation of sterol regulatory element-binding protein 1 (SREBP1) and suppresses lipogenesis in cancer cells," Oncogene, vol. 35, no. 5, pp. 642-650, 2016.

[17] Y.-K. Seo, H. K. Chong, A. M. Infante, S.-S. Im, X. Xie, and T. F. Osborne, "Genome-wide analysis of SREBP-1 binding in mouse liver chromatin reveals a preference for promoter proximal binding to a new motif," Proceedings of the National Academy of Sciences, vol. 106, no. 33, pp. 13765-13769, 2009.

[18] T. S. van Kempen, M. H. Wenink, E. F. A. Leijten, T. R. D. J. Radstake, and M. Boes, "Perception of self: distinguishing autoimmunity from autoinflammation," Nature Reviews Rheumatology, vol. 11, no. 8, pp. 483-492, 2015.

[19] Y. Oishi, N. J. Spann, V. M. Link et al., "SREBP1 contributes to resolution of pro-inflammatory TLR4 signaling by reprogramming fatty acid metabolism," Cell Metabolism, vol. 25, no. 2, pp. 412-427, 2017.

[20] A. Yaron, F. Naider, and S. Scharpe, "Proline-dependent structural and biological properties of peptides and proteins," Critical Reviews in Biochemistry and Molecular Biology, vol. 28, no. 1, pp. 31-81, 1993.

[21] D. J. Drucker, "The role of gut hormones in glucose homeostasis," Journal of Clinical Investigation, vol. 117, no. 1, pp. 24-32, 2007.

[22] M. H. A. Muskiet, M. M. Smits, L. M. Morsink, and M. Diamant, "The gut-renal axis: do incretin-based agents confer renoprotection in diabetes?," Nature Reviews Nephrology, vol. 10, no. 2, pp. 88-103, 2014.

[23] T. Tanaka, D. Camerini, B. Seed et al., "Cloning and functional expression of the T cell activation antigen CD 26," Journal of Immunology, vol. 149, no. 2, pp. 481-486, 1992.

[24] K. Rufinatscha, B. Radlinger, J. Dobner et al., "Dipeptidyl peptidase-4 impairs insulin signaling and promotes lipid accumulation in hepatocytes," Biochemical and Biophysical Research Communications, vol. 485, no. 2, pp. 366-371, 2017.

[25] E. E. Mulvihill, "Dipeptidyl peptidase inhibitor therapy in type 2 diabetes: control of the incretin axis and regulation of postprandial glucose and lipid metabolism," Peptides, vol. 100, pp. 158-164, 2018.

[26] N. Matikainen, S. Mänttäri, A. Schweizer et al., "Vildagliptin therapy reduces postprandial intestinal triglyceride-rich lipoprotein particles in patients with type 2 diabetes," Diabetologia, vol. 49, no. 9, pp. 2049-2057, 2006.

[27] W. Yano, N. Inoue, S. Ito et al., "Mechanism of lipid-lowering action of the dipeptidyl peptidase- 4 inhibitor, anagliptin, in low-density lipoprotein receptor-deficient mice," Journal of Diabetes Investigation, vol. 8, no. 2, pp. 155-160, 2017.

[28] H. Motohashi and M. Yamamoto, "Nrf2-Keap1 defines a physiologically important stress response mechanism," Trends in Molecular Medicine, vol. 10, no. 11, pp. 549-557, 2004.

[29] N. Nagata, L. Xu, S. Kohno et al., "Glucoraphanin ameliorates obesity and insulin resistance through adipose tissue browning and reduction of metabolic endotoxemia in mice," Diabetes, vol. 66, no. 5, pp. 1222-1236, 2017.

[30] M. Begley, C. G. M. Gahan, and C. Hill, "The interaction between bacteria and bile," FEMS Microbiology Reviews, vol. 29, no. 4, pp. 625-651, 2005. 
[31] S. A. Joyce, J. MacSharry, P. G. Casey et al., "Regulation of host weight gain and lipid metabolism by bacterial bile acid modification in the gut," Proceedings of the National Academy of Sciences, vol. 111, no. 20, pp. 7421-7426, 2014.

[32] A. Everard, V. Lazarevic, M. Derrien et al., "Responses of gut microbiota and glucose and lipid metabolism to prebiotics in genetic obese and diet-induced leptin-resistant mice," Diabetes, vol. 60, no. 11, pp. 2775-2786, 2011.

[33] M. Serino, E. Luche, S. Gres et al., "Metabolic adaptation to a high-fat diet is associated with a change in the gut microbiota," Gut, vol. 61, no. 4, pp. 543-553, 2012.

[34] A. Everard, C. Belzer, L. Geurts et al., "Cross-talk between Akkermansia muciniphila and intestinal epithelium controls diet-induced obesity," Proceedings of the National Academy of Sciences, vol. 110, no. 22, pp. 9066-9071, 2013.

[35] D. Porras, E. Nistal, S. Martínez-Flórez et al., "Protective effect of quercetin on high-fat diet-induced non-alcoholic fatty liver disease in mice is mediated by modulating intestinal microbiota imbalance and related gut-liver axis activation," Free Radical Biology and Medicine, vol. 102, pp. 188202, 2017.

[36] M. Schneeberger, A. Everard, A. G. Gómez-Valadés et al., "Akkermansia muciniphila inversely correlates with the onset of inflammation, altered adipose tissue metabolism and metabolic disorders during obesity in mice," Scientific Reports, vol. 5, no. 1, 2015.

[37] T. Q. de Aguiar Vallim, E. J. Tarling, and P. A. Edwards, "Pleiotropic roles of bile acids in metabolism," Cell Metabolism, vol. 17, no. 5, pp. 657-669, 2013.

[38] A. J. Cox, N. P. West, and A. W. Cripps, "Obesity, inflammation, and the gut microbiota," The Lancet Diabetes \& Endocrinology, vol. 3, no. 3, pp. 207-215, 2015.

[39] J. Fernandes, W. Su, S. Rahat-Rozenbloom, T. M. S. Wolever, and E. M. Comelli, "Adiposity, gut microbiota and faecal short chain fatty acids are linked in adult humans," Nutrition \& Diabetes, vol. 4, no. 6, article e121, 2014.

[40] Y. C. Chen, G. H. Dong, K. C. Lin, and Y. L. Lee, "Gender difference of childhood overweight and obesity in predicting the risk of incident asthma: a systematic review and meta-analysis," Obesity Reviews, vol. 14, no. 3, pp. 222231, 2013.

[41] U. Frey, P. Latzin, J. Usemann, J. Maccora, U. Zumsteg, and S. Kriemler, "Asthma and obesity in children: current evidence and potential systems biology approaches," Allergy, vol. 70, no. 1, pp. 26-40, 2015.

[42] R. Granell, A. J. Henderson, D. M. Evans et al., "Effects of BMI, fat mass, and lean mass on asthma in childhood: a Mendelian randomization study," PLoS Medicine, vol. 11, no. 7, article e1001669, 2014.

[43] R. Barros, P. Moreira, P. Padrão et al., "Obesity increases the prevalence and the incidence of asthma and worsens asthma severity," Clinical Nutrition, vol. 36, no. 4, pp. 1068-1074, 2017.

[44] T. Hampton, "Studies probe links between childhood asthma and obesity," JAMA, vol. 311, no. 17, pp. 1718-1719, 2014.

[45] L. P. Boulet, "Asthma and obesity," Clinical \& Experimental Allergy, vol. 43, no. 1, pp. 8-21, 2013.

[46] F. M. Wensveen, S. Valentić, M. Šestan, T. Turk Wensveen, and B. Polić, "The "Big Bang" in obese fat: events initiating obesity-induced adipose tissue inflammation," European Journal of Immunology, vol. 45, no. 9, pp. 2446-2456, 2015.
[47] R. Medzhitov, "Origin and physiological roles of inflammation," Nature, vol. 454, no. 7203, pp. 428-435, 2008.

[48] H. Tashiro, K. Takahashi, H. Sadamatsu et al., "Saturated fatty acid increases lung macrophages and augments house dust mite-induced airway inflammation in mice fed with high-fat diet," Inflammation, vol. 40, no. 3, pp. 1072-1086, 2017.

[49] G. Yu, L. Zhu, H. Li et al., "Influence of gender on OVAinduced airway inflammation in C57/B6J mice on a high-fat diet," European Journal of Inflammation, vol. 16, 2018.

[50] H. A. Periyalil, L. G. Wood, T. A. Wright et al., "Obese asthmatics are characterized by altered adipose tissue macrophage activation," Clinical \& Experimental Allergy, 2018.

[51] C. N. Lumeng, S. M. DeYoung, J. L. Bodzin, and A. R. Saltiel, "Increased inflammatory properties of adipose tissue macrophages recruited during diet-induced obesity," Diabetes, vol. 56, no. 1, pp. 16-23, 2006.

[52] Y. Tian, L. Gao, Y. Guo, and Y. Xu, "Short-term phlorizin treatment attenuates adipose tissue inflammation without alerting obesity in high-fat diet fed mice," Journal of Food Biochemistry, vol. 41, no. 6, 2017.

[53] C. Crewe, Y. A. An, and P. E. Scherer, "The ominous triad of adipose tissue dysfunction: inflammation, fibrosis, and impaired angiogenesis," Journal of Clinical Investigation, vol. 127, no. 1, pp. 74-82, 2017.

[54] S. Legrand-Poels, N. Esser, L. L'homme, A. Scheen, N. Paquot, and J. Piette, "Free fatty acids as modulators of the NLRP3 inflammasome in obesity/type 2 diabetes," Biochemical Pharmacology, vol. 92, no. 1, pp. 131-141, 2014.

[55] C. Brightling, M. Berry, and Y. Amrani, "Targeting TNF- $\alpha$ : a novel therapeutic approach for asthma," Journal of Allergy and Clinical Immunology, vol. 121, no. 1, pp. 5-10, 2008.

[56] M. Chehimi, H. Vidal, and A. Eljaafari, "Pathogenic role of IL-17-producing immune cells in obesity, and related inflammatory diseases," Journal of Clinical Medicine, vol. 6, no. 7, p. 68, 2017.

[57] E. D. Telenga, S. W. Tideman, H. A. M. Kerstjens et al., "Obesity in asthma: more neutrophilic inflammation as a possible explanation for a reduced treatment response," Allergy, vol. 67, no. 8, pp. 1060-1068, 2012.

[58] O. Sideleva, K. Black, and A. E. Dixon, "Effects of obesity and weight loss on airway physiology and inflammation in asthma," Pulmonary Pharmacology \& Therapeutics, vol. 26, no. 4, pp. 455-458, 2013.

[59] D.-V. Nguyen, A. Linderholm, A. Haczku, and N. Kenyon, "Glucagon-like peptide 1: a potential anti-inflammatory pathway in obesity-related asthma," Pharmacology \& Therapeutics, vol. 180, pp. 139-143, 2017.

[60] V. Apostolopoulos, M. P. J. de Courten, L. Stojanovska, G. L. Blatch, K. Tangalakis, and B. de Courten, "The complex immunological and inflammatory network of adipose tissue in obesity," Molecular Nutrition \& Food Research, vol. 60, no. 1, pp. 43-57, 2016.

[61] S. Talukdar, D. Y. Oh, G. Bandyopadhyay et al., "Neutrophils mediate insulin resistance in mice fed a high-fat diet through secreted elastase," Nature Medicine, vol. 18, no. 9, pp. 14071412, 2012.

[62] T. Y. F. Halim, C. A. Steer, L. Mathä et al., "Group 2 innate lymphoid cells are critical for the initiation of adaptive $\mathrm{T}$ helper 2 cell-mediated allergic lung inflammation," Immunity, vol. 40, no. 3, pp. 425-435, 2014. 
[63] Y. Zhou, X. Yu, H. Chen et al., "Leptin deficiency shifts mast cells toward anti-inflammatory actions and protects mice from obesity and diabetes by polarizing M2 macrophages," Cell Metabolism, vol. 22, no. 6, pp. 1045-1058, 2015.

[64] M. J. Hubler and A. J. Kennedy, "Role of lipids in the metabolism and activation of immune cells," The Journal of Nutritional Biochemistry, vol. 34, pp. 1-7, 2016.

[65] S. A. Shore, "Mechanistic basis for obesity-related increases in ozone-induced airway hyperresponsiveness in mice," Annals of the American Thoracic Society, vol. 14, Supplement_5, pp. S357-S362, 2017.

[66] L. Maggi, G. Montaini, A. Mazzoni et al., "Human circulating group 2 innate lymphoid cells can express CD154 and promote IgE production," Journal of Allergy and Clinical Immunology, vol. 139, no. 3, pp. 964-976.e4, 2017.

[67] F. Huang, B. E. del-Río-Navarro, S. Torres-Alcántara et al., "Adipokines, asymmetrical dimethylarginine, and pulmonary function in adolescents with asthma and obesity," Journal of Asthma, vol. 54, no. 2, pp. 153-161, 2016.

[68] S. Galic, J. S. Oakhill, and G. R. Steinberg, "Adipose tissue as an endocrine organ," Molecular and Cellular Endocrinology, vol. 316, no. 2, pp. 129-139, 2010.

[69] C. Nowak, J. Sundström, S. Gustafsson et al., "Protein biomarkers for insulin resistance and type 2 diabetes risk in two large community cohorts," Diabetes, vol. 65, no. 1, pp. 276-284, 2016.

[70] H.-S. Moon, G. Matarese, A. M. Brennan et al., "Efficacy of metreleptin in obese patients with type 2 diabetes: cellular and molecular pathways underlying leptin tolerance," Diabetes, vol. 60, no. 6, pp. 1647-1656, 2011.

[71] K. J. Hasenkrug, "The leptin connection: regulatory T cells and autoimmunity," Immunity, vol. 26, no. 2, pp. 143-145, 2007.

[72] X.-M. Mai, Y. Chen, and D. Krewski, "Does leptin play a role in obesity-asthma relationship?," Pediatric Allergy and Immunology, vol. 20, no. 3, pp. 207-212, 2009.

[73] H. Zheng, X. Zhang, E. F. Castillo, Y. Luo, M. Liu, and X. O. Yang, "Leptin enhances TH2 and ILC2 responses in allergic airway disease," Journal of Biological Chemistry, vol. 291, no. 42, pp. 22043-22052, 2016.

[74] M. Kubo, "Innate and adaptive type 2 immunity in lung allergic inflammation,” Immunological Reviews, vol. 278, no. 1, pp. 162-172, 2017.

[75] W. Hao, J. Wang, Y. Zhang, Y. Wang, L. Sun, and W. Han, "Leptin positively regulates MUC5AC production and secretion induced by interleukin-13 in human bronchial epithelial cells," Biochemical and Biophysical Research Communications, vol. 493, no. 2, pp. 979-984, 2017.

[76] S. S. Martinez, A. Campa, R. Marlink et al., "Higher leptin levels are associated with obesity and slower HIV disease progression," The FASEB Journal, vol. 31, no. 1, pp. 964981, 2017.

[77] A. Salah, M. Ragab, W. Mansour, and M. Taher, "Leptin and adiponectin are valuable serum markers explaining obesity/ bronchial asthma interrelationship," Egyptian Journal of Chest Diseases and Tuberculosis, vol. 64, no. 3, pp. 529-533, 2015.

[78] G. Fantuzzi, "Adiponectin and inflammation: consensus and controversy," Journal of Allergy and Clinical Immunology, vol. 121, no. 2, pp. 326-330, 2008.
[79] M. Elfeky, R. Kaede, Y. Okamatsu-Ogura, and K. Kimura, "Adiponectin inhibits LPS-induced HMGB1 release through an AMP kinase and heme oxygenase-1-dependent pathway in RAW 264 macrophage cells," Mediators of Inflammation, vol. 2016, 9 pages, 2016.

[80] Y. Luo and M. Liu, "Adiponectin: a versatile player of innate immunity," Journal of Molecular Cell Biology, vol. 8, no. 2, pp. 120-128, 2016.

[81] Y. Hayashikawa, M. Iwata, M. Inomata et al., "Association of serum adiponectin with asthma and pulmonary function in the Japanese population," Endocrine Journal, vol. 62, no. 8, pp. 695-709, 2015.

[82] M. Bednarska-Makaruk, A. Graban, A. Wiśniewska et al., "Association of adiponectin, leptin and resistin with inflammatory markers and obesity in dementia," Biogerontology, vol. 18, no. 4, pp. 561-580, 2017.

[83] H. M. Al-Asy and M. M. Mabrouk, "Serum resistin as an asthma marker and predictor of inhaled corticosteroid response in bronchial asthma in children," Egyptian Journal of Chest Diseases and Tuberculosis, vol. 66, no. 3, pp. 391394, 2017.

[84] J. Gray, K. Oehrle, G. Worthen, T. Alenghat, J. Whitsett, and H. Deshmukh, "Intestinal commensal bacteria mediate lung mucosal immunity and promote resistance of newborn mice to infection," Science Translational Medicine, vol. 9, no. 376, 2017.

[85] S. Tavares da Silva, C. Araújo dos Santos, and J. Bressan, "Intestinal microbiota; relevance to obesity and modulation by prebiotics and probiotics," Nutricion Hospitalaria, vol. 28, no. 4, pp. 1039-1048, 2013.

[86] R. Caesar, V. Tremaroli, P. Kovatcheva-Datchary, P. D. Cani, and F. Bäckhed, "Crosstalk between gut microbiota and dietary lipids aggravates wat inflammation through tlr signaling," Cell Metabolism, vol. 22, no. 4, pp. 658$668,2015$.

[87] K. Tuomi and J. V. Logomarsino, "Bacterial lipopolysaccharide, lipopolysaccharide-binding protein, and other inflammatory markers in obesity and after bariatric surgery," Metabolic Syndrome and Related Disorders, vol. 14, no. 6, pp. 279-288, 2016.

[88] R. Ahmad, M. F. Sorrell, S. K. Batra, P. Dhawan, and A. B. Singh, "Gut permeability and mucosal inflammation: bad, good or context dependent," Mucosal Immunology, vol. 10, no. 2, pp. 307-317, 2017.

[89] L. Catrysse and G. van Loo, "Inflammation and the metabolic syndrome: the tissue-specific functions of NF- $\kappa \mathrm{B}$," Trends in Cell Biology, vol. 27, no. 6, pp. 417-429, 2017.

[90] A. Trompette, E. S. Gollwitzer, K. Yadava et al., "Gut microbiota metabolism of dietary fiber influences allergic airway disease and hematopoiesis," Nature Medicine, vol. 20, no. 2, pp. 159-166, 2014.

[91] P. M. Smith, M. R. Howitt, N. Panikov et al., "The microbial metabolites, short-chain fatty acids, regulate colonic Treg cell homeostasis," Science, vol. 341, no. 6145, pp. 569-573, 2013.

[92] S. Steinmeyer, K. Lee, A. Jayaraman, and R. C. Alaniz, "Microbiota metabolite regulation of host immune homeostasis: a mechanistic missing link," Current Allergy and Asthma Reports, vol. 15, no. 5, p. 24, 2015.

[93] J. L. Sonnenburg and F. Bäckhed, "Diet-microbiota interactions as moderators of human metabolism," Nature, vol. 535, no. 7610, pp. 56-64, 2016. 
[94] S. N. Heinritz, E. Weiss, M. Eklund et al., "Intestinal microbiota and microbial metabolites are changed in a pig model fed a high-fat/low-fiber or a low-fat/high-fiber diet," PLoS One, vol. 11, no. 4, article e0154329, 2016.

[95] G. Tolhurst, H. Heffron, Y. S. Lam et al., "Short-chain fatty acids stimulate glucagon-like peptide-1 secretion via the Gprotein-coupled receptor FFAR2," Diabetes, vol. 61, no. 2, pp. 364-371, 2012.

[96] A. J. D. Barros, L. P. Santos, F. Wehrmeister et al., "Caesarean section and adiposity at 6,18 and 30 years of age: results from three Pelotas (Brazil) birth cohorts," BMC Public Health, vol. 17, no. 1, pp. 256-259, 2017.

[97] M. G. Dominguez-Bello, E. K. Costello, M. Contreras et al., "Delivery mode shapes the acquisition and structure of the initial microbiota across multiple body habitats in newborns," Proceedings of the National Academy of Sciences, vol. 107, no. 26, pp. 11971-11975, 2010.

[98] K. A. Martinez II, J. C. Devlin, C. R. Lacher et al., "Increased weight gain by $\mathrm{C}$-section: functional significance of the primordial microbiome," Science Advances, vol. 3, no. 10, 2017.

[99] L.-J. Qian, S.-M. Kang, J.-L. Xie et al., "Early-life gut microbial colonization shapes Th1/Th2 balance in asthma model in BALB/c mice," BMC Microbiology, vol. 17, no. 1, p. 135, 2017.

[100] B. Aryal, A. K. Singh, N. Rotllan, N. Price, and C. Fernández-Hernando, "MicroRNAs and lipid metabolism," Current Opinion in Lipidology, vol. 28, no. 3, pp. 273-280, 2017.

[101] T. Horie, T. Nishino, O. Baba et al., "MicroRNA-33 regulates sterol regulatory element-binding protein 1 expression in mice," Nature Communications, vol. 4, no. 1, p. 2883, 2013.

[102] V. Rottiers and A. M. Näär, "MicroRNAs in metabolism and metabolic disorders," Nature Reviews Molecular Cell Biology, vol. 13, no. 4, pp. 239-250, 2012.

[103] J. Horton, J. Cohen, and H. Hobbs, "Molecular biology of PCSK9: its role in LDL metabolism," Trends in Biochemical Sciences, vol. 32, no. 2, pp. 71-77, 2007.

[104] M. S. Sabatine, R. P. Giugliano, S. D. Wiviott et al., "Efficacy and safety of evolocumab in reducing lipids and cardiovascular events," New England Journal of Medicine, vol. 372, no. 16, pp. 1500-1509, 2015.

[105] J. J. Tang, J. G. Li, W. Qi et al., "Inhibition of SREBP by a small molecule, betulin, improves hyperlipidemia and insulin resistance and reduces atherosclerotic plaques," Cell Metabolism, vol. 13, no. 1, pp. 44-56, 2011.

[106] S. S. Shin and M. Yoon, "Korean red ginseng (Panax ginseng) inhibits obesity and improves lipid metabolism in high fat diet-fed castrated mice," Journal of Ethnopharmacology, vol. 210, pp. 80-87, 2018.

[107] S. Woo, M. Yoon, J. Kim et al., "The anti-angiogenic herbal extract from Melissa officinalis inhibits adipogenesis in 3T3$\mathrm{L} 1$ adipocytes and suppresses adipocyte hypertrophy in high fat diet-induced obese C57BL/6J mice," Journal of Ethnopharmacology, vol. 178, pp. 238-250, 2016.

[108] F. M. Siraj, S. Natarajan, M. A. Huq, Y. J. Kim, and D. C. Yang, "Structural investigation of ginsenoside Rf with PPAR $\gamma$ major transcriptional factor of adipogenesis and its impact on adipocyte," Journal of Ginseng Research, vol. 39, no. 2, pp. 141-147, 2015.

[109] J.-W. Yang and S. Kim, "Ginsenoside Rc promotes antiadipogenic activity on 3T3-L1 adipocytes by down- regulating $\mathrm{C} / \mathrm{EBP} \alpha$ and $\operatorname{PPAR} \gamma$, , Molecules, vol. 20, no. 1, pp. 1293-1303, 2015.

[110] J. Zhong, X. Rao, J. Deiuliis et al., “A potential role for dendritic cell/macrophage-expressing DPP4 in obesity-induced visceral inflammation," Diabetes, vol. 62, no. 1, pp. 149$157,2012$.

[111] F. Zhuge, Y. Ni, M. Nagashimada et al., "DPP-4 inhibition by linagliptin attenuates obesity-related inflammation and insulin resistance by regulating M1/M2 macrophage polarization," Diabetes, vol. 65, no. 10, pp. 2966-2979, 2016.

[112] Y. Ni, M. Nagashimada, F. Zhuge et al., "Astaxanthin prevents and reverses diet-induced insulin resistance and steatohepatitis in mice: a comparison with vitamin E," Scientific Reports, vol. 5, no. 1, 2015.

[113] Y. Ni, M. Nagashimada, L. Zhan et al., "Prevention and reversal of lipotoxicity-induced hepatic insulin resistance and steatohepatitis in mice by an antioxidant carotenoid, $\beta$-cryptoxanthin," Endocrinology, vol. 156, no. 3, pp. 987999, 2015.

[114] Z. Yu, W. Shao, Y. Chiang et al., "Oltipraz upregulates the nuclear respiratory factor 2 alpha subunit (NRF2) antioxidant system and prevents insulin resistance and obesity induced by a high-fat diet in C57BL/6J mice," Diabetologia, vol. 54, no. 4, pp. 922-934, 2011.

[115] S. Shin, J. Wakabayashi, M. S. Yates et al., "Role of Nrf2 in prevention of high-fat diet-induced obesity by synthetic triterpenoid CDDO-imidazolide," European Journal of Pharmacology, vol. 620, no. 1-3, pp. 138-144, 2009.

[116] D. de Zeeuw, T. Akizawa, P. Audhya et al., "Bardoxolone methyl in type 2 diabetes and stage 4 chronic kidney disease," New England Journal of Medicine, vol. 369, no. 26, pp. 24922503, 2013.

[117] G. Musso, R. Gambino, and M. Cassader, "Gut microbiota as a regulator of energy homeostasis and ectopic fat deposition: mechanisms and implications for metabolic disorders," Current Opinion in Lipidology, vol. 21, no. 1, pp. 76-83, 2010.

[118] Y. Cho and S. A. Shore, "Obesity, asthma, and the microbiome," Physiology, vol. 31, no. 2, pp. 108-116, 2016.

[119] X. Li, Y. Song, X. Ma et al., "Lactobacillus plantarum and Lactobacillus fermentum alone or in combination regulate intestinal flora composition and systemic immunity to alleviate obesity syndrome in high-fat diet rat," International Journal of Food Science \& Technology, vol. 53, no. 1, pp. 137-146, 2018.

[120] R. Liu, J. Hong, X. Xu et al., "Gut microbiome and serum metabolome alterations in obesity and after weight-loss intervention," Nature Medicine, vol. 23, no. 7, pp. 859-868, 2017.

[121] Y. Li, R. Tang, P. S. C. Leung, M. E. Gershwin, and X. Ma, "Bile acids and intestinal microbiota in autoimmune cholestatic liver diseases," Autoimmunity Reviews, vol. 16, no. 9, pp. 885-896, 2017.

[122] Y. Kusumoto, J. Irie, K. Iwabu et al., "Bile acid binding resin prevents fat accumulation through intestinal microbiota in high-fat diet-induced obesity in mice," Metabolism, vol. 71, pp. 1-6, 2017

[123] Y. Guo, Y. C. Zhang, W. H. Huang, F. P. Selwyn, and C. D. Klaassen, "Dose-response effect of berberine on bile acid profile and gut microbiota in mice," BMC Complementary and Alternative Medicine, vol. 16, no. 1, p. 394, 2016. 
[124] R. Sun, N. Yang, B. Kong et al., "Orally administered berberine modulates hepatic lipid metabolism by altering microbial bile acid metabolism and the intestinal FXR signaling pathway," Molecular Pharmacology, vol. 91, no. 2, pp. 110-122, 2017.

[125] G. M. Pasinetti, L. M. Ho, J. M. Faith, and K. M. Ono, "Role of gut microbiota-derived polyphenolic acid in attenuation of protein misfolding in neurodegeneration," The FASEB Journal, vol. 31, no. 1, 2017.

[126] M. A. Martinez-Gonzalez, C. Sayon-Orea, M. Ruiz-Canela, C. de la Fuente, A. Gea, and M. Bes-Rastrollo, "Yogurt consumption, weight change and risk of overweight/obesity: the SUN cohort study," Nutrition, Metabolism and Cardiovascular Diseases, vol. 24, no. 11, pp. 1189-1196, 2014.

[127] A. Yeganeh, P. Zahradka, and C. G. Taylor, "Trans-10,cis-12 conjugated linoleic acid (t10-c12 CLA) treatment and caloric restriction differentially affect adipocyte cell turnover in obese and lean mice," The Journal of Nutritional Biochemistry, vol. 49, pp. 123-132, 2017.

[128] P. Prabhala, "The Impact of Precise MKP-1 Regulation and Modulation on Cytokine Expression in Asthma and Airway Remodeling," Postgraduate Theses, 2016.

[129] M. F. Gregor and G. S. Hotamisligil, "Inflammatory mechanisms in obesity," Annual Review of Immunology, vol. 29, no. 1, pp. 415-445, 2011.

[130] N. Hu and Y. Zhang, "TLR4 knockout attenuated high fat diet-induced cardiac dysfunction via NF- $\kappa \mathrm{B} / \mathrm{JNK}$-dependent activation of autophagy," Biochimica et Biophysica Acta (BBA) - Molecular Basis of Disease, vol. 1863, no. 8, pp. 2001-2011, 2017.

[131] J. H. C. Yiu, B. Dorweiler, and C. W. Woo, "Interaction between gut microbiota and toll-like receptor: from immunity to metabolism," Journal of Molecular Medicine, vol. 95, no. 1, pp. 13-20, 2017.

[132] C. Y. Li, S. R. Erickson, and C. H. Wu, "Metformin use and asthma outcomes among patients with concurrent asthma and diabetes," Respirology, vol. 21, no. 7, pp. 1210-1218, 2016.

[133] M. Gauthier, A. Ray, and S. E. Wenzel, "Evolving concepts of asthma," American Journal of Respiratory and Critical Care Medicine, vol. 192, no. 6, pp. 660-668, 2015.

[134] H. G. Ortega, M. C. Liu, I. D. Pavord et al., "Mepolizumab treatment in patients with severe eosinophilic asthma," New England Journal of Medicine, vol. 371, no. 13, pp. 11981207, 2014.

[135] M. Castro, J. Zangrilli, M. E. Wechsler et al., "Reslizumab for inadequately controlled asthma with elevated blood eosinophil counts: results from two multicentre, parallel, doubleblind, randomised, placebo-controlled, phase 3 trials," The Lancet Respiratory Medicine, vol. 3, no. 5, pp. 355-366, 2015.

[136] V. Ramirez-Carrozzi, A. Sambandam, M. Zhou et al., "Combined blockade of the IL-13 and IL-33 pathways leads to a greater inhibition of type 2 inflammation over inhibition of either pathway alone," Journal of Allergy and Clinical Immunology, vol. 139, no. 2, pp. 705-708.e6, 2017.

[137] J. Corren, R. F. Lemanske Jr, N. A. Hanania et al., "Lebrikizumab treatment in adults with asthma," New England Journal of Medicine, vol. 365, no. 12, pp. 1088-1098, 2011.

[138] N. A. Hanania, M. Noonan, J. Corren et al., "Lebrikizumab in moderate-to-severe asthma: pooled data from two randomised placebo-controlled studies," Thorax, vol. 70, no. 8, pp. 748-756, 2015.

[139] A. D. Parulekar, C. C. Kao, Z. Diamant, and N. A. Hanania, "Targeting the interleukin-4 and interleukin-13 pathways in severe asthma: current knowledge and future needs," Current Opinion in Pulmonary Medicine, vol. 24, no. 1, pp. 50-55, 2018.

[140] T. A. Doherty, "At the bench: understanding group 2 innate lymphoid cells in disease," Journal of Leukocyte Biology, vol. 97, no. 3, pp. 455-467, 2015.

[141] D. Rigas, G. Lewis, J. L. Aron et al., “Type 2 innate lymphoid cell suppression by regulatory $\mathrm{T}$ cells attenuates airway hyperreactivity and requires inducible $\mathrm{T}$-cell costimulatorinducible T-cell costimulator ligand interaction," Journal of Allergy and Clinical Immunology, vol. 139, no. 5, pp. 14681477.e2, 2017.

[142] H. Y. Kim, H. J. Lee, Y.-J. Chang et al., "Interleukin-17-producing innate lymphoid cells and the NLRP3 inflammasome facilitate obesity-associated airway hyperreactivity," Nature Medicine, vol. 20, no. 1, pp. 54-61, 2014.

[143] S. E. Wenzel, "Asthma phenotypes: the evolution from clinical to molecular approaches," Nature Medicine, vol. 18, no. 5, pp. 716-725, 2012.

[144] M. L. Hernandez, K. Mills, M. Almond et al., "IL-1 receptor antagonist reduces endotoxin-induced airway inflammation in healthy volunteers," Journal of Allergy and Clinical Immunology, vol. 135, no. 2, pp. 379-385, 2015.

[145] M. C. Calixto, L. Lintomen, D. M. André et al., "Metformin attenuates the exacerbation of the allergic eosinophilic inflammation in high fat-diet-induced obesity in mice," PLoS One, vol. 8, no. 10, article e76786, 2013.

[146] G. M. Gauvreau, P. M. O'Byrne, L.-P. Boulet et al., "Effects of an anti-TSLP antibody on allergen-induced asthmatic responses," New England Journal of Medicine, vol. 370, no. 22, pp. 2102-2110, 2014.

[147] J. Liu, R. Sakurai, and V. K. Rehan, "PPAR- $\gamma$ agonist rosiglitazone reverses perinatal nicotine exposure-induced asthma in rat offspring," American Journal of Physiology-Lung Cellular and Molecular Physiology, vol. 308, no. 8, pp. L788-L796, 2015.

[148] Y.-S. Yoon, S.-Y. Kim, M.-J. Kim, J.-H. Lim, M.-S. Cho, and J. L. Kang, "PPAR $\gamma$ activation following apoptotic cell instillation promotes resolution of lung inflammation and fibrosis via regulation of efferocytosis and proresolving cytokines," Mucosal Immunology, vol. 8, no. 5, pp. 1031-1046, 2015.

[149] J. Dib, A. Thomas, P. Delahaut, E. Fichant, W. Schänzer, and M. Thevis, "Identification and characterization of in vitro and in vivo generated metabolites of the adiponectin receptor agonists AdipoRon and 112254," Journal of Pharmaceutical and Biomedical Analysis, vol. 125, pp. 68-76, 2016.

[150] A. Engin, "Adiponectin-resistance in obesity," Advances in Experimental Medicine and Biology, vol. 960, pp. 415-441, 2017. 


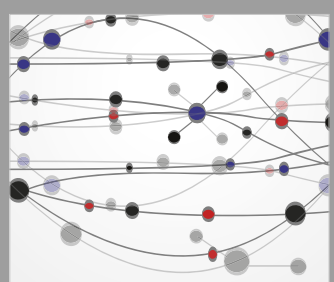

The Scientific World Journal
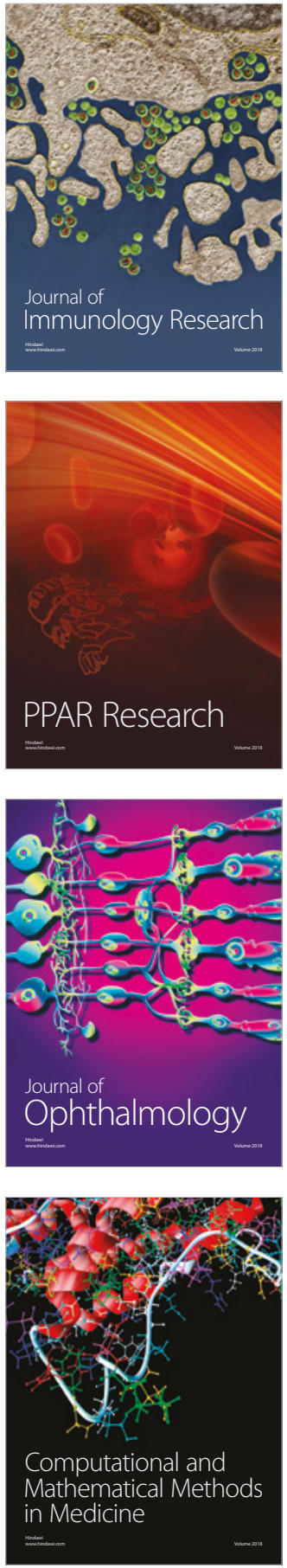

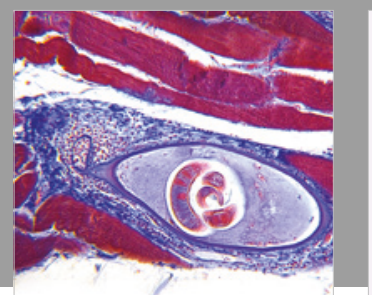

Gastroenterology Research and Practice

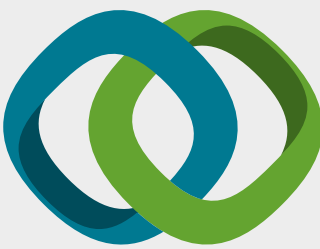

\section{Hindawi}

Submit your manuscripts at

www.hindawi.com
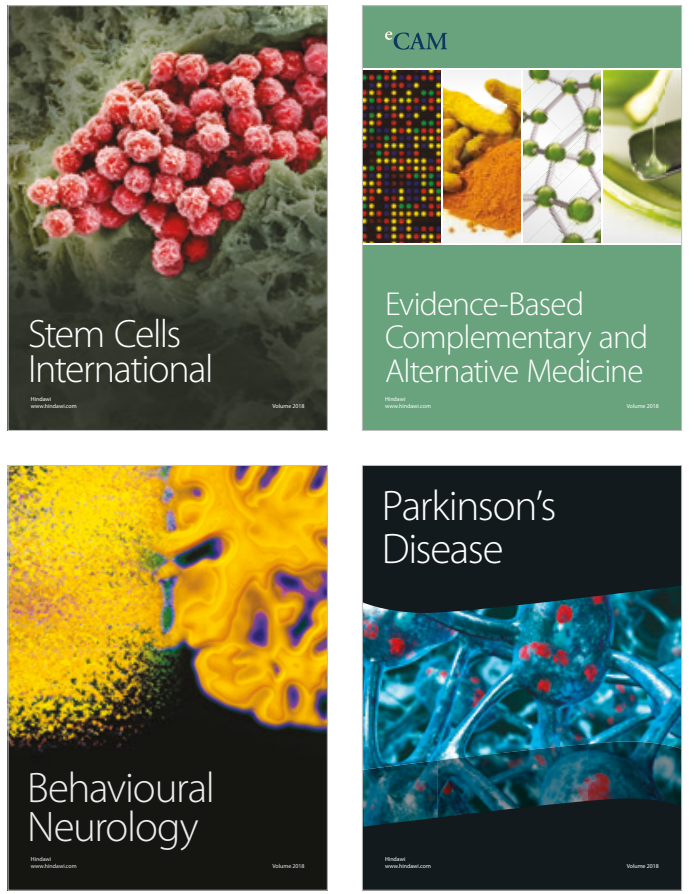

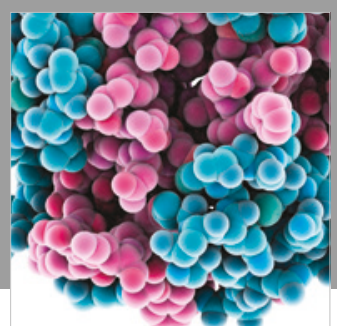

ournal of

Diabetes Research

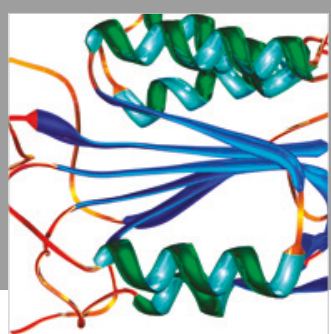

Disease Markers
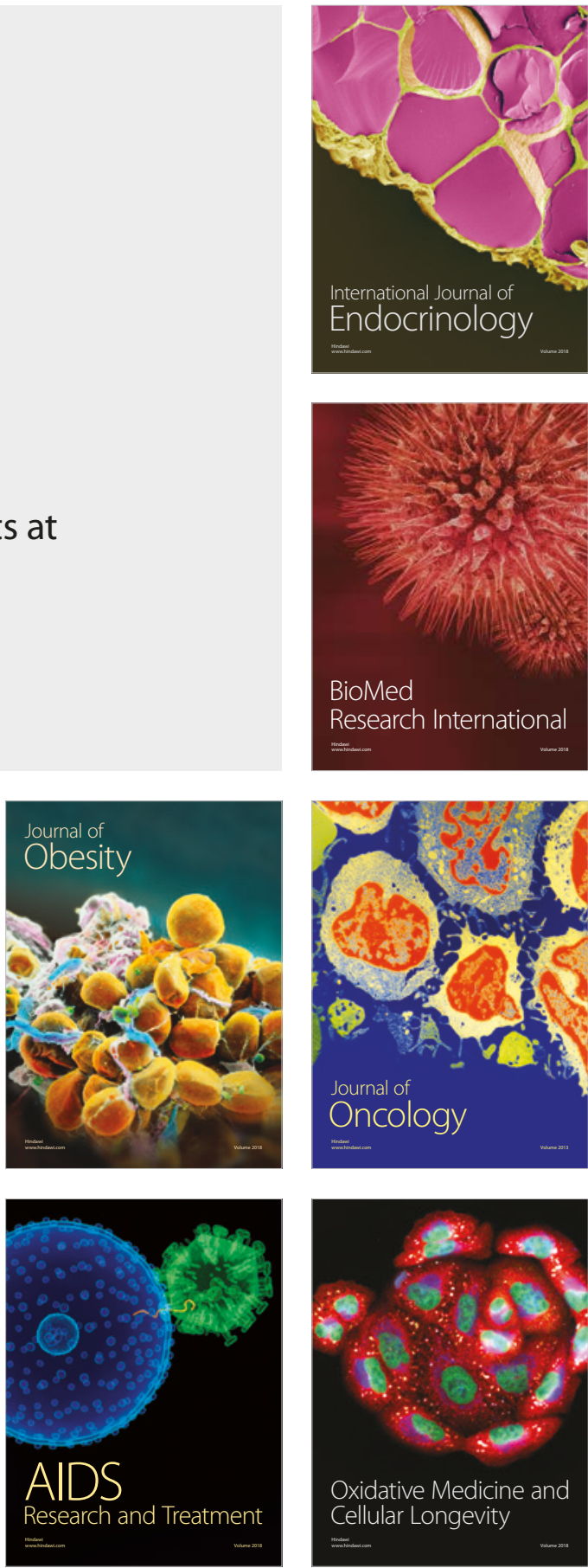